\title{
CONSTRUCTION AND ANALYSIS OF PROBABILITY MODELS FOR CONTROLLED EVOLUTIONARY SYSTEMS
}

UDC 519.21

\author{
M. A. FEDOTKIN
}

\begin{abstract}
Constructing adequate probability models is a basic problem in studies of controlled evolutionary systems. Three approaches are known nowadays to construct models of such systems. There are some disadvantages of the existing approaches and another approach, based on cybernetics, is proposed. Some features of the cybernetic approach are demonstrated in this paper by solving the well-known independence paradox.
\end{abstract}

\section{Solution of a Mosteller problem by a Classical approach}

In this section, we illustrate the construction of the Kolomogorov probability model for statistically stable experiments with control by solving a Mosteller problem [4].

Mosteller problem. To encourage the son's promising tennis carrer, his father offers him a prize if he wins at least two tennis sets in a row in a three-set series to be played with his father and the club champion alternately. The club champion is a better player than the father. The son wins against the club champion with probability $p$ and against the father with probability $q>p$. The son can choose between the following two series: 1) champion, father, champion; 2) father, champion, father. Which series should the son choose in order to maximize the probability to win the prize?

To check whether the basic conclusions of the solution to the Mosteller problem are adequate [4, 5], one should repeat the tennis match of the son vs. the father and the club champion many times. This experiment cannot be carried out easily. Therefore, instead of tennis matches, let us consider a similar experiment that has been repeated many times by the author during examinations at different faculties of the N. I. Lobachevsky State University of Nizhniy Novgorod.

Exams problem. Students take an exam in probability theory consisting in alternately answering three questions to the professor and an assistant professor The students know that the first and the third questions deal with the theory and the second question is to solve a practical problem. A student passes the exam if he gives at least two positive

2010 Mathematics Subject Classification. Primary 60K99, 93C99.

Key words and phrases. Controlled evolutionary systems, probability model, cybernetic approach, independence paradox.

This work was done within the research project "Analysis of discrete managing service systems and systems for calculation of Boole functions", \# 0120.0602598, of N. I. Lobachevsky State University of Nizhni Novgorod.

The paper is based on a talk delivered at the International Conference "Modern Stochastics: Theory and Applications II", held at the Taras Shevchenko Kyiv National University on September 7-11, 2010, and is dedicated to anniversaries of the prominent Ukrainian scientists: Anatoly Skorokhod, Vladimir Korolyuk, and Igor Kovalenko. 
answers in a row. A student gives a correct answer to the professor with probability $p$ and does the same to the assistant professor with probability $q>p$. A student is asked to choose between one of two sequences of answering the questions: 1) professorassistant professor-professor ( $\mathrm{P}-\mathrm{AP}-\mathrm{P})$, or 2$)$ assistant professor-professor-assistant professor (AP-P-AP). Let us consider the core of the solution of this instructive problem which was proposed in [4, 5].

Suppose that $A_{1}, A_{2}, A_{3}$ denote the events that a student succeeds in answering the first question to the professor, the second question to the assistant professor, the third question to the professor, respectively, and let $A$ stand for the event that a student passes the exam under behavior pattern $\mathrm{P}-\mathrm{AP}-\mathrm{P}$. We introduce similar notation for the behavior pattern AP-P-AP, namely let $B_{1}, B_{2}$, and $B_{3}$ denote the events that a student succeeds in answering the first question to the assistant professor, the second question to the professor, the third question to the assistant professor, respectively, and let $B$ stand for the event that a student passes the exam under behavior pattern $\mathrm{AP}-\mathrm{P}-\mathrm{AP}$. Then

$$
A=\left(A_{1} \cap A_{2}\right) \cup\left(A_{2} \cap A_{3}\right), \quad B=\left(B_{1} \cap B_{2}\right) \cup\left(B_{2} \cap B_{3}\right) .
$$

Denote by $\mathbf{P}_{1}(\cdot)$ the probability measure given that the student behaves according to the sequence $\mathrm{P}-\mathrm{AP}-\mathrm{P}$, and by $\mathbf{P}_{0}(\cdot)$ the probability measure given that the student behaves according to the sequence $\mathrm{AP}-\mathrm{P}-\mathrm{AP}$. If answers to different teachers are independent, then

$$
\begin{aligned}
& \mathbf{P}_{1}(A)=\mathbf{P}_{1}\left(A_{1} \cap A_{2}\right)+\mathbf{P}_{1}\left(A_{2} \cap A_{3}\right)-\mathbf{P}_{1}\left(A_{1} \cap A_{2} \cap A_{3}\right)=p q+q p-p q p, \\
& \mathbf{P}_{0}(B)=\mathbf{P}_{0}\left(B_{1} \cap B_{2}\right)+\mathbf{P}_{0}\left(B_{2} \cap B_{3}\right)-\mathbf{P}_{0}\left(B_{1} \cap B_{2} \cap B_{3}\right)=q p+p q-q p q .
\end{aligned}
$$

This gives $\mathbf{P}_{1}(A)>\mathbf{P}_{0}(B)$ if $q>p$. Therefore the students should behave according to sequence 1).

Mosteller [4] justifies this conclusion by saying that a student's capacity to solve applied problems is more important. In other words, the relation $\mathbf{P}_{1}\left(A_{2}\right)=q>\mathbf{P}_{0}\left(B_{2}\right)=p$ is more important for passing the exam than the difficulty in having to face the professor twice in the sequence $\mathrm{P}-\mathrm{AP}-\mathrm{P}$.

Székely states in [5] that Mosteller's solution of the problem and the conclusions are strongly based on the assumption that the answers are independent.

The author's statistical evidence shows, however, that the students who were taking exams between 1967 and 1992 were choosing the sequence P-AP-P. From 1992 on, they prefer the sequence $\mathrm{AP}-\mathrm{P}-\mathrm{AP}$, which seems to them more reasonable. In order to explain this behavior, we first give a formal solution to the problem using a classical approach based on the "black box" principle, and then we apply a non-traditional approach [1, 2, 3] to the study of stochastic experiments with control.

It is well known 1 that one of the aims in probability theory consists in constructing an adequate Kolmogorov model $\left(\Omega, \mathfrak{F}, \mathbf{P}_{r}(\cdot)\right)$ of a statistically stable stochastic experiment $W_{r}$ under control $r$ belonging to a set $R$. An arbitrary element $\omega$ in $\Omega$ corresponds to the so-called elementary outcome of the stochastic experiment $W_{r}$. The collection $\mathfrak{F}$ of subsets of an a priori given set of all possible outcomes constitutes a $\sigma$-algebra and contains all observable outcomes $D \subset \Omega$ of the experiment $W_{r}$. Finally, a probability function of the form $\mathbf{P}_{r}(\cdot): \mathfrak{F} \rightarrow[0,1]$ is defined on the $\sigma$-algebra $\mathfrak{F}$ and depends on the given control $r \in R$.

If the "black box" approach is used, then a statistically stable stochastic experiment $W_{r}$ is interpreted as a control object $E_{r}$ and a control system $C_{r}$. In this case, we assume that an elementary outcome of the experiment $W_{r}$ is defined uniquely, following a certain law from the results of observation and carrying out elementary outcomes of each of the experiments $E_{r}$ and $C_{r}$. Therefore, the experiment $W_{r}$ is an ordered pair 
$\left(E_{r}, C_{r}\right)$ and we will write this fact as $W_{r}=\left(E_{r}, C_{r}\right)$. Assume that the maps $\varphi_{r}(\omega)$ and $\psi_{r}(\omega)$ measure the outcome of the control object $E_{r}$ and that of the control system $C_{r}$, while the functions $\Phi\left(\varphi_{r}(\omega), \psi_{r}(\omega)\right)$ describe the gain of the experiment $W_{r}$ from control $r$. If under control $r$ the expected income is $\mathrm{M}_{r} \Phi\left(\varphi_{r}(\omega), \psi_{r}(\omega)\right)$, then the objective of the experiment $W_{r}$ is to apply an optimal control $r_{0} \in R$ satisfying

$$
\mathrm{M}_{r_{0}} \Phi\left(\varphi_{r_{0}}(\omega), \psi_{r_{0}}(\omega)\right)=\sup \left\{\mathrm{M}_{r} \Phi\left(\varphi_{r}(\omega), \psi_{r}(\omega)\right): r \in R\right\}
$$

Assume now that in the exams problem that the control object $E_{r}$ is successive student's answers to questions one, two, and three, and the control system $C_{r}$ corresponds to one of the two behavior patterns (controls) chosen by the student. Here, $r=1$ if the first behavior pattern is chosen and $r=0$ otherwise. This implies that $R=\{0,1\}$. Note that, in this problem, the results of the control object $E_{r}$ do not affect those of the control system $C_{r}$. Then, in fact, the contents of the Mosteller solution to the exams problem is based on the representation of the experiment $W_{r}$ as the control system $\left(E_{r}, C_{r}\right)$ having no feedback channel. This situation is represented in Figure 1 .

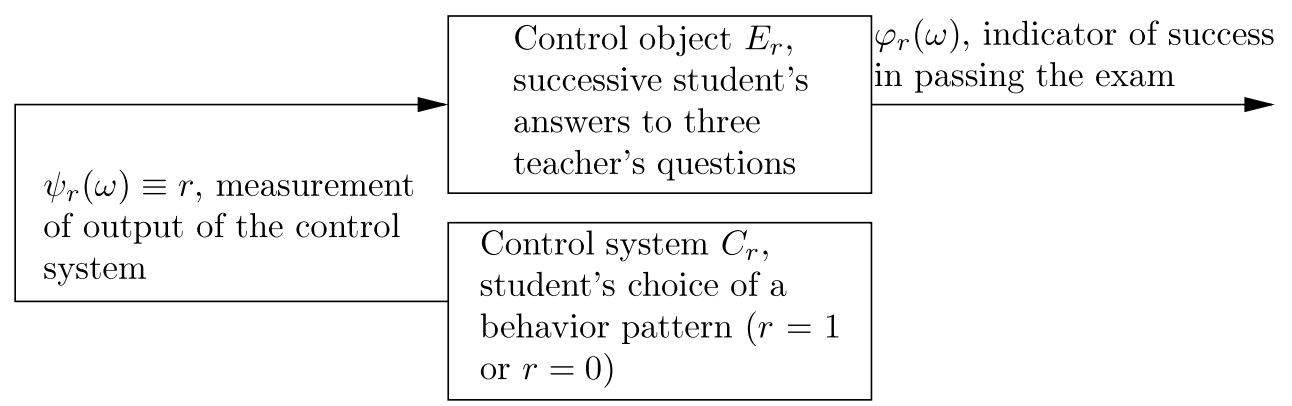

FiguRE 1. A model for the experiment of exams having no feedback channel

In Figure 1, the quantities $\varphi_{r}(\omega)$ and $\psi_{r}(\omega)$ measure the elementary outcome of the experiment $W_{r}$ according to the sequence of answers of the student to the instructors (control object $E_{r}$ ) and according to the student choice of the behavior pattern (control system $C_{r}$ ), respectively. Here, the value of the random variable $\varphi_{r}(\omega)$ equals one if the student passes the exam and equals zero otherwise, while the value of the random variable $\psi_{r}(\omega)$ equals the control chosen, $r=0,1$. For this experiment, we have $\Phi\left(\varphi_{r}(\omega), \psi_{r}(\omega)\right) \equiv$ $\varphi_{r}(\omega)$.

The following assertion holds for this model.

Lemma 1. The optimal control is $r_{0}=1$. This means that a student should choose the strategy $P-A P-P$.

Proof. One can choose the probability space $\left(\Omega, \mathfrak{F}, \mathbf{P}_{r}(\cdot)\right)$ as a model of the experiment $W_{r}$, where $\Omega=\left\{\omega=\left(u_{1}, u_{2}, u_{3}\right): u_{1}, u_{2}, u_{3}=0,1\right\}$ and $\mathfrak{F}=\{D: D \subset \Omega\}$. For $i=1,2$, 3 we put $u_{i}=1$ if a student gives a correct answer to question $i$, and $u_{i}=0$ otherwise. For example, the event $\{(1,1,1)\}$ corresponds to correct answers to all three questions, while the event $\{(0,0,1)\}$ means that a student has given a correct answer only to the third question. Since students' answers are independent, we have

$$
\mathbf{P}_{r}(\{(1,1,1)\})=p q p, \quad \mathbf{P}_{r}(\{(0,0,1)\})=(1-p)(1-q) p
$$

if $r=1$, and

$$
\mathbf{P}_{r}(\{(1,1,1)\})=q p q, \quad \mathbf{P}_{r}(\{(0,0,1)\})=(1-q)(1-p) q
$$


if $r=0$. If $A$ stands for the event that a student passes the exam, then

$$
A=\{(1,1,0),(0,1,1),(1,1,1)\}
$$

and

$$
\mathbf{P}_{r}(A)=\mathbf{P}_{r}(\{(1,1,0)\})+\mathbf{P}_{r}(\{(0,1,1)\})+\mathbf{P}_{r}(\{(1,1,1)\})=r p q(q-p)+p q(2-q) .
$$

Since $\varphi_{r}(\omega)$ is the indicator of the random event $A$, we have $M_{r} \Phi\left(\varphi_{r}(\omega), \psi_{r}(\omega)\right)=\mathbf{P}_{r}(A)$. By the inequality $q>p$ and by the optimality condition

$$
\mathrm{M}_{r_{0}} \Phi\left(\varphi_{r_{0}}(\omega), \psi_{r_{0}}(\omega)\right)=\sup \left\{\mathrm{M}_{r} \Phi\left(\varphi_{r}(\omega), \psi_{r}(\omega)\right): r=0,1\right\}
$$

we conclude that the optimal control is $r_{0}=1$. That is, the strategy $\mathrm{P}-\mathrm{AP}-\mathrm{P}$ is more favorable for the student.

\section{MostelleR's PROBlem AS A CYBERnETIC CONTROL SYSTEM}

The approach presented in Section 1 is in fact based on a formal description of the relationship between input $\psi_{r}(\omega)$ and output $\varphi_{r}(\omega)$ of the control object $E_{r}$. This relationship is given by the formula

$$
\mathbf{P}_{r}\left(\left\{\omega: \varphi_{r}(\omega)=1\right\}\right)=r p q(q-p)+p q(2-q)
$$

defining the distribution of the random variable $\varphi_{r}(\omega): \Omega \rightarrow\{0,1\}$. In other words, the approach presented above studies a statistically stable experiment $W_{r}=\left(E_{r}, C_{r}\right)$ with control $r \in R$ by using the "black box" principle as it is used in problems of automatic control. The main drawback of this approach is that the random variables $\psi_{r}(\omega)$ and $\varphi_{r}(\omega)$ in fact are only a convenient mathematical model for measuring inputs and outputs of the control object $E_{r}$, but not a probability model of the statistically stable experiment $W_{r}$ on the whole. Therefore the "black box" principle which is simple and structurally accessible does not require a detailed study of the experiment $W_{r}$. Therefore it does not permit us to use both the deterministic and stochastic natures of the experiment $W_{r}$ to their full extent.

When one studies a real-life control system, there are difficult questions regarding an effective description of this system, as well as the design of the study of this system taking into account its physical nature and functional objectives. Therefore it is useful to look at a real-life system not from the point of view of a "black box," but rather from a view that accounts for the general fundamental properties of the system and the methodological concept of a control system given in mathematical cybernetics [6]. This approach was essentially supported and consistently developed in [1, 2, 3. The following fundamental statements form the basis of the construction, analysis, and optimization of a model of a control system:

(a) any control system possesses such properties as scheme, information, coordinates, and function;

(b) the principle of discrete functioning of a control system at times $\tau_{i}, i \geqslant 0$, where the point stochastic process $\left\{\tau_{i} ; i \geqslant 0\right\}$, defines the scale of time encompassed on the halfline $[0, \infty)$;

(c) the principle of construction of a scheme from the following structural blocks: environment, input and output poles, internal and external memories, information processing devices for internal and external memories.

Under this non-traditional approach, the construction of a probability model for a control system includes the following basic steps.

(1) Isolating the scheme, the information, the coordinates and the function of a system.

(2) Defining the so-called structural blocks for the scheme. 
(3) Determining the algorithms that control changes in the states of the structural blocks.

(4) Codifying the information or a non-local description of the structural blocks.

(5) Revealing functional and statistical links between system blocks.

(6) Choosing the main basic and target characteristics of the system.

(7) Solving the problems of the analysis, synthesis, and optimization of the control system.

Consider now an application of the cybernetic approach to the solution of the independence paradox [4, 5] in the Mosteller problem [4]. The Mosteller problem, or the exams problem, can adequately be represented by means of the control system whose organizational chart is represented in Figure 2,

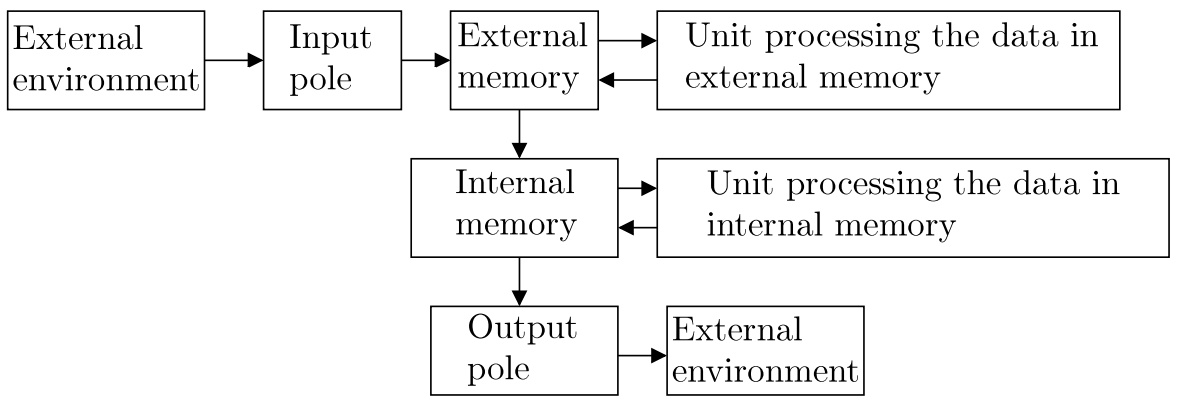

FiguRE 2. Scheme of a control system for the exams problem

Let us reveal the non-formal meaning of all structural blocks of the control system corresponding to the exams problem. For this purpose, denote by $\tau_{0}$ the time when a student gets his examination sheet containing three questions and when he declares his behavior according to pattern $\mathrm{P}-\mathrm{AP}-\mathrm{P}$, with probability $r \in[0,1]$, or according to pattern $\mathrm{AP}-\mathrm{P}-\mathrm{AP}$, with probability $1-r$. Furthermore, let $\tau_{i}$ be the time when the student completes his answer to the question $i$, where $i=1,2,3$. We consider a general framework of the problem where we do not assume that students' answers to different questions need not be independent under any behavior strategy. We will first provide in item 2.1 a mathematical description and properties of the environment and then, in items 2.2 2.7, we will give a mathematical description and properties of all remaining blocks of the scheme.

2.1. The processes creating the environment take a long time and are conservative. The environment consists of conditions before the exam that suggest that a student chooses his behavior strategy among two available patterns. This implies that the environment has the following two states: 1) $\mathrm{P}-\mathrm{AP}-\mathrm{P}$ denoted by $b_{1}$, and 2) $\mathrm{AP}-\mathrm{P}-\mathrm{AP}$ denoted $b_{2}$.

Assume that the environment at the time $\tau_{0}$ takes value $b_{1}$ with probability $r \in[0,1]$ and takes value $b_{2}$ with probability $1-r$. In other words, the student chooses the behavior pattern $\mathrm{P}-\mathrm{AP}-\mathrm{P}$ with probability $r$ and the pattern $\mathrm{AP}-\mathrm{P}-\mathrm{AP}$ with probability $1-r$. The information provided by the environment is the set $\{\mathrm{P}-\mathrm{AP}-\mathrm{P}, \mathrm{AP}-\mathrm{P}-\mathrm{AP}\}=\left\{b_{1}, b_{2}\right\}$ containing two states of the environment. The coordinates of the environment are the numbers 1 and 2 corresponding to its states $b_{1}$ and $b_{2}$, respectively. The state of the environment at the time $\tau_{i}$ is a random element $\chi_{i} \equiv \chi_{0}$ for $i=0,1,2,3$. The information code of the environment is the random vector $\chi=\left(\chi_{0}, \chi_{1}, \chi_{2}, \chi_{3}\right)$. Here, the numbers $\mathbf{P}\left(\chi_{0}=b_{1}\right)=r, \mathbf{P}\left(\chi_{0}=b_{2}\right)=1-r$ of the random element $\chi_{0}$ determines the probability distribution of the random vector $\chi$ and gives the following transition graph of the states of the environment: 


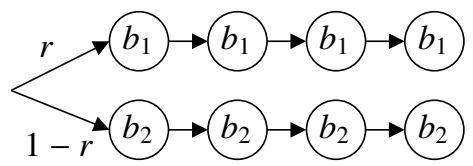

FiguRE 3. Transition graph of the environment

2.2. An input pole means that a student obtains at time $\tau_{0}$ an examination sheet containing questions $w_{1}, w_{2}$, and $w_{3}$. The information in the input pole is the set $\left\{\left(w_{1}, w_{2}, w_{3}\right)\right\}$ containing the one and only state $\left(w_{1}, w_{2}, w_{3}\right)$ of the input pole. The vector $\alpha_{i} \equiv\left(w_{1}, w_{2}, w_{3}\right)$ defines the state of the input pole at time $\tau_{i}$ for $i=0,1,2,3$. The information code of the input pole is the vector $\alpha=\left(\alpha_{0}, \alpha_{1}, \alpha_{2}, \alpha_{3}\right)$ whose components are equal. Therefore the directed graph of state transitions of the input pole can be represented as in Figure 4

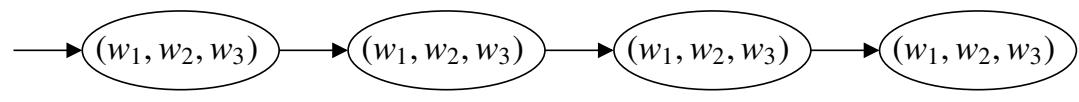

Figure 4. Graph of state transitions of the input pole

2.3. The external memory registers the questions to be answered by a student. The information in the external memory is the set $\left\{\left(w_{1}, w_{2}, w_{3}\right),\left(w_{2}, w_{3}\right), w_{3}, \varnothing\right\}$ containing four states of the external memory: $d_{1}=\left(w_{1}, w_{2}, w_{3}\right), d_{2}=\left(w_{2}, w_{3}\right), d_{3}=w_{3}$ and $d_{4}=\varnothing$. The coordinates of the external memory are the numbers $1,2,3$, and 4 of its states $d_{1}, d_{2}, d_{3}$, and $d_{4}$, respectively. The state of the external memory at time $\tau_{i}$ is $\beta_{i}=d_{i+1}$ for $i=0,1,2,3$. The information code of the external memory is the vector $\beta=\left(\beta_{0}, \beta_{1}, \beta_{2}, \beta_{3}\right)$. This implies that the directed graph of the state transitions of the external memory can be represented as in Figure 5.

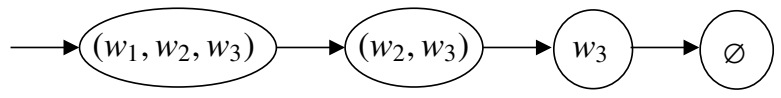

FIGURE 5. Graph of state transitions of the external memory

2.4. The unit which processes information in the external memory deletes in a deterministic way all questions a student has already answered. In this way, it changes the state number for the external memory in the sequence $1,2,3$, and 4 . The information in the external memory processing unit is the set $\left\{\varnothing, w_{1},\left(w_{1}, w_{2}\right),\left(w_{1}, w_{2}, w_{3}\right)\right\}$ containing four states $e_{1}=\varnothing, e_{2}=w_{1}, e_{3}=\left(w_{1}, w_{2}\right)$, and $e_{4}=\left(w_{1}, w_{2}, w_{3}\right)$ of this unit. The coordinates of the external memory processing unit are the numbers $1,2,3$, and 4 corresponding to the states $e_{1}, e_{2}, e_{3}$, and $e_{4}$ of the unit, respectively. The state of the external memory processing unit at time $\tau_{i}$ is $\zeta_{i}=e_{i+1}, i=0,1,2,3$. The information code of the external memory processing unit is the vector $\zeta=\left(\zeta_{0}, \zeta_{1}, \zeta_{2}, \zeta_{3}\right)$, and the directed graph of state transitions of this unit is represented in Figure 6 .

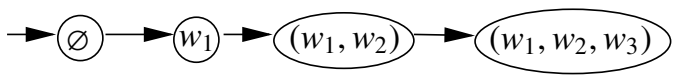

FiguRE 6. Graph of state transitions of the external memory processing unit 
2.5. The internal memory registers the behavior pattern of the student and records the sequence of the three answers to the instructors. The information in the internal memory unit is the set having four internal memory states $f_{1}, f_{2}, f_{3}$, and $f_{4}$, where

$\left(f_{1}\right)$ the student has chosen the first behavior pattern,

$\left(f_{2}\right)$ the student has chosen the second behavior pattern,

$\left(f_{3}\right)$ the student has answered a question,

$\left(f_{4}\right)$ the student has failed to answer a question.

The coordinates of the internal memory are the numbers $1,2,3$, and 4 of the states $f_{1}, f_{2}, f_{3}$, and $f_{4}$, respectively. The state of the internal memory at time $\tau_{i}$ is the random element $\eta_{i}$ with $i=0,1,2,3$. Here, $\eta_{0} \in\left\{f_{1}, f_{2}\right\}, \eta_{1} \in\left\{f_{3}, f_{4}\right\}, \eta_{2} \in\left\{f_{3}, f_{4}\right\}$, and $\eta_{3} \in\left\{f_{3}, f_{4}\right\}$. The information code of the internal memory is the random vector $\eta=\left(\eta_{0}, \eta_{1}, \eta_{2}, \eta_{3}\right)$. The directed graph of the state transitions of the internal memory unit is represented in Figure 7 .

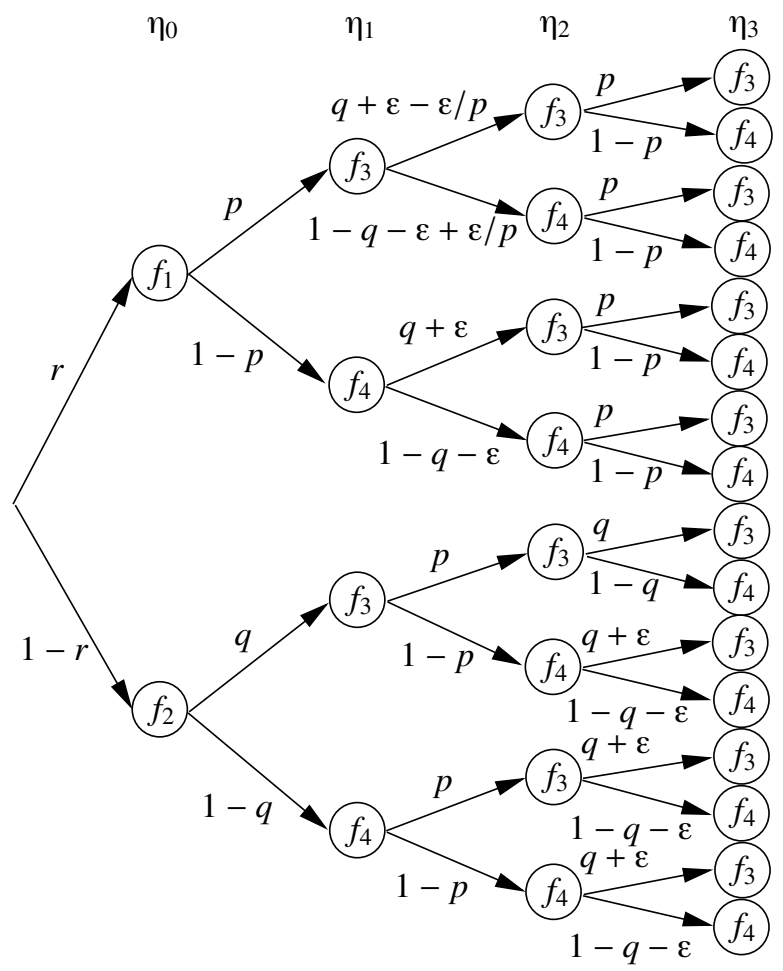

FiguRE 7. Graph of state transitions of the internal memory unit

Recall that, according to the assumptions of the exams problem, a student answers questions sequentially and, in general, the answers are dependent. Given $\varepsilon \geqslant 0$, this dependence is determined by the conditional distributions of the random elements $\eta_{0}$, 
$\eta_{1}, \eta_{2}$, and $\eta_{3}$ as given below:

$$
\begin{aligned}
& \mathbf{P}\left(\eta_{0}=f_{1}\right)=r, \quad \mathbf{P}\left(\eta_{1}=f_{3} \mid \eta_{0}=f_{1}\right)=p, \quad \mathbf{P}\left(\eta_{1}=f_{3} \mid \eta_{0}=f_{2}\right)=q, \\
& \mathbf{P}\left(\eta_{2}=f_{3} \mid \eta_{0}=f_{1}, \eta_{1}=f_{3}\right)=q+\varepsilon-\varepsilon / p \geqslant p, \\
& \mathbf{P}\left(\eta_{2}=f_{3} \mid \eta_{0}=f_{1}, \eta_{1}=f_{4}\right)=\mathbf{P}\left(\eta_{3}=f_{3} \mid \eta_{0}=f_{2}, \eta_{1}=f_{3}, \eta_{2}=f_{4}\right) \\
& =\mathbf{P}\left(\eta_{3}=f_{3} \mid \eta_{0}=f_{2}, \eta_{1}=f_{4}, \eta_{2}=f_{4}\right)=q+\varepsilon<1, \\
& \mathbf{P}\left(\eta_{3}=f_{3} \mid \eta_{0}=f_{2}, \eta_{1}=f_{3}, \eta_{2}=f_{3}\right)=q, \\
& \mathbf{P}\left(\eta_{2}=f_{3} \mid \eta_{0}=f_{2}, \eta_{1}=f_{3}\right)=\mathbf{P}\left(\eta_{2}=f_{3} \mid \eta_{0}=f_{2}, \eta_{1}=f_{4}\right) \\
& =\mathbf{P}\left(\eta_{3}=f_{3} \mid \eta_{0}=f_{1}, \eta_{1}=f_{3}, \eta_{2}=f_{3}\right) \\
& =\mathbf{P}\left(\eta_{3}=f_{3} \mid \eta_{0}=f_{1}, \eta_{1}=f_{3}, \eta_{2}=f_{4}\right) \\
& =\mathbf{P}\left(\eta_{3}=f_{3} \mid \eta_{0}=f_{1}, \eta_{1}=f_{4}, \eta_{2}=f_{3}\right) \\
& =\mathbf{P}\left(\eta_{3}=f_{3} \mid \eta_{0}=f_{1}, \eta_{1}=f_{4}, \eta_{2}=f_{4}\right)=p \text {. }
\end{aligned}
$$

These equalities correspond to the graph of the state transitions of the internal memory unit. Let us give a different interpretation of equalities (1)-(5) for the conditional probabilities that describe the dependence in the sequence of student's answers.

Equality (11) follows immediately from the assumptions of the Mosteller problem, since the mechanism employed by the student from choosing the behavior pattern is random.

Equality (2) shows that, for a student who is not well prepared, the probability of a correct answer to the assistant professor after a successful answer to the professor decreases. This circumstance can be justified by the argument that the student gets more psychological self-confidence after a successful answer to the professor.

Equality (3) means that, for a student who is not well prepared, the probability of answer to the assistant professor after an unsuccessful answer to the professor increases by $\varepsilon$. This fact is illustrated in a large number of observations when the students whose preparation is weak try to be more careful and answer the next question more thoroughly after an unsuccessful answer. On the other hand, highly qualified instructors often give a "last chance" to students whose preparation is weak and who fail to answer some questions. In this way, a highly qualified and experienced instructor educates the student.

Equality (41) corresponds to a quite natural situation where the answer of a student to the third question does not depend on his correct answers to the first two questions.

Finally, the group of equalities (5) gives evidence that the professor evaluates students' skills more strictly and, at the same time, in a more objective way than the assistant professor. In other words, a professor's assessment of a student does not depend on previous, successful or unsuccessful, answers of the student. This simple algorithm suggests a model for educating students during exams who do not have an adequate preparation.

Furthermore we consider the internal memory processing unit which carries out this educational model for weakly prepared students explained above.

2.6. The internal memory processing unit determines, by some algorithm, the conditional probabilities of a student's next successful answer depending on his previous answers and carries out a random mechanism of assessment of the answers. The information in the internal memory processing unit is the set

$$
\{r,(p, q),(q+\varepsilon-\varepsilon / p, q+\varepsilon, p, p),(p, p, p, p, q, q+\varepsilon, q+\varepsilon, q+\varepsilon)\}
$$

containing the following four states:

$$
\begin{gathered}
g_{1}=r, \quad g_{2}=(p, q), \quad g_{3}=(q+\varepsilon-\varepsilon / p, q+\varepsilon, p, p), \\
g_{4}=(p, p, p, p, q, q+\varepsilon, q+\varepsilon, q+\varepsilon) .
\end{gathered}
$$


The coordinates of the internal memory processing unit are the numbers $1,2,3$, and 4 of the states $g_{1}, g_{2}, g_{3}$, and $g_{4}$, respectively. The state of the unit at time $\tau_{i}$ is $\theta_{i}=g_{i+1}$ for $i=0,1,2,3$. The information code of the unit is the vector $\theta=\left(\theta_{0}, \theta_{1}, \theta_{2}, \theta_{3}\right)$. Therefore the directed graph of state transitions of this unit has a simple form represented in Figure 8 .

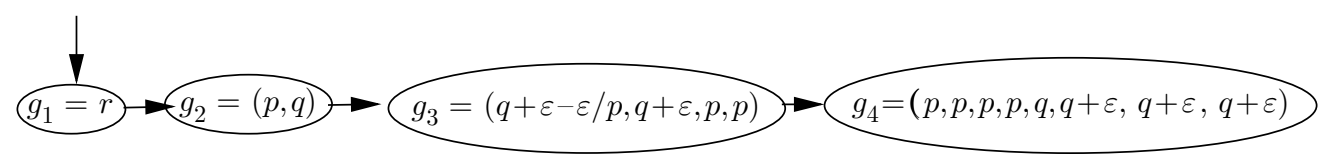

FiguRE 8. Graph of state transitions of the internal memory processing unit

2.7. The output pole defines the outcomes that can be observed after a student's exam. The information of the output pole unit corresponding to the experiment under consideration is the space

$$
\Omega=\left\{\omega=\left(x_{0}, x_{1}, x_{2}, x_{3}\right): x_{0} \in\left\{f_{1}, f_{2}\right\}, x_{1}, x_{2}, x_{3} \in\left\{f_{3}, f_{4}\right\}\right\}
$$

containing the following 16 states:

$$
\begin{array}{rll}
\omega_{1}=\left(f_{1}, f_{3}, f_{3}, f_{3}\right), & \omega_{2}=\left(f_{1}, f_{3}, f_{3}, f_{4}\right), & \omega_{3}=\left(f_{1}, f_{3}, f_{4}, f_{3}\right), \\
\omega_{4}=\left(f_{1}, f_{3}, f_{4}, f_{4}\right), & \omega_{5}=\left(f_{1}, f_{4}, f_{3}, f_{3}\right), & \omega_{6}=\left(f_{1}, f_{4}, f_{3}, f_{4}\right), \\
\omega_{7}=\left(f_{1}, f_{4}, f_{4}, f_{3}\right), & \omega_{8}=\left(f_{1}, f_{4}, f_{4}, f_{4}\right), & \omega_{9}=\left(f_{2}, f_{3}, f_{3}, f_{3}\right), \\
\omega_{10}=\left(f_{2}, f_{3}, f_{3}, f_{4}\right), & \omega_{11}=\left(f_{2}, f_{3}, f_{4}, f_{3}\right), & \omega_{12}=\left(f_{2}, f_{3}, f_{4}, f_{4}\right), \\
\omega_{13}=\left(f_{2}, f_{4}, f_{3}, f_{3}\right), & \omega_{14}=\left(f_{2}, f_{4}, f_{3}, f_{4}\right), & \omega_{15}=\left(f_{2}, f_{4}, f_{4}, f_{3}\right), \\
& \omega_{16}=\left(f_{2}, f_{4}, f_{4}, f_{4}\right) . &
\end{array}
$$

The coordinates of the output pole unit are the numbers $1,2, \ldots$, and 16 of the states

$$
\omega_{1}, \omega_{2}, \ldots, \text { and } \omega_{16},
$$

respectively.

The state of such a unit at time $\tau_{3}$ is a random element $\xi$ where $\xi(\omega) \equiv \omega$ for $\omega=$ $\left(x_{0}, x_{1}, x_{2}, x_{3}\right) \in \Omega$. The information code of the output pole unit is the random vector $\xi=\left(\xi_{0}, \xi_{1}, \xi_{2}, \xi_{3}\right)$ where $\xi_{i}(\omega) \equiv x_{i}$ for any $i=0, \ldots, 3$.

According to equalities (10)-(5) and using the graph of state transitions of the internal memory, a directed graph of appearances of states of the output pole can be constructed. This graph is depicted in Figure 9 below.

Figure 9 also shows the probabilities of appearances of different states of the output pole. The symbol $A$ is used to mark the states $\omega_{1}, \omega_{2}, \omega_{5}, \omega_{9}, \omega_{10}$ and $\omega_{13}$ of the output pole where the student passes the exam.

The results in [1, 2, 3] show that the cybernetic approach enables us not only to find functional and statistical links between system's units, but also to choose effectively main starting and target characteristics of the control system in order to solve the problem of analysis and optimization.

This statement also holds for the Mosteller problem. Indeed, except for the environmental unit, internal memory unit, and output pole, the behavior of all remaining units is either deterministic or is defined uniquely by the states of other units. Therefore one can reconstruct completely the behavior of the control system of the examination process by only observations of random states of the output pole unit at time $\tau_{3}$. In particular, one can determine all states of the output pole unit when the student passes the exam. Therefore, the state of the output pole unit can be taken as the basic characteristics of 


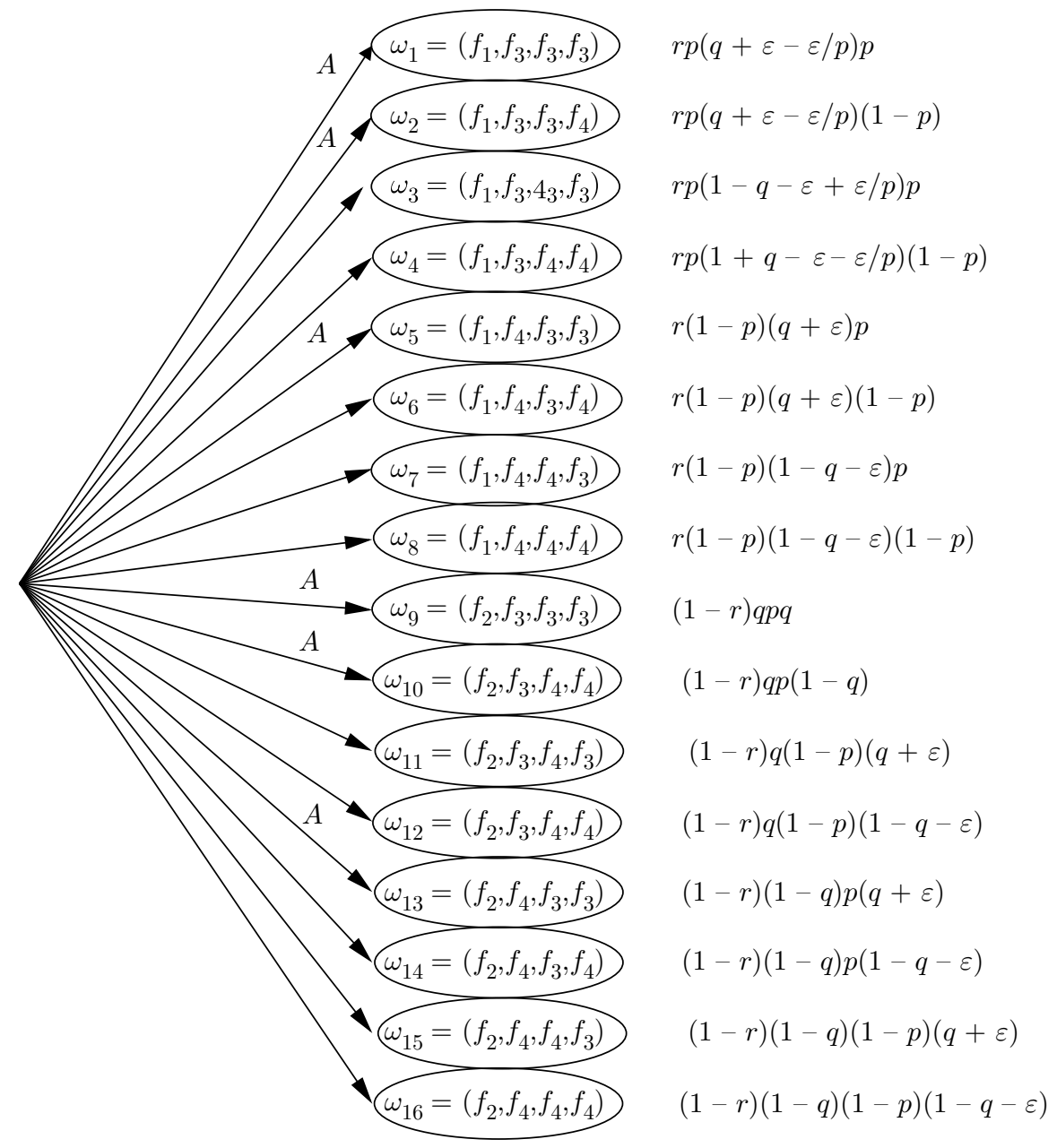

FiguRE 9. Graph of emerging states for the output pole unit

the exams problem. In this situation, the output pole unit models the operation of the statistically stable experiment $W_{r}$, while each state of the output pole unit is identified with a description of an elementary outcome of the experiment $W_{r}$.

At this point, it is easy to construct the Kolmogorov probability model $\left(\Omega, \mathfrak{F}, \mathbf{P}_{r}(\cdot)\right)$ of the experiment $W_{r}$. Here, the certain event is $\Omega=\left\{\omega_{1}, \omega_{2}, \ldots, \omega_{16}\right\}$, the $\sigma$-algebra is $\mathfrak{F}=\{B: B \subset \Omega\}=\left\{B_{1}, B_{2}, \ldots, B_{65536}\right\}$, and the probability $\mathbf{P}_{r}(\cdot)$ is given by the following equalities:

$$
\begin{aligned}
\mathbf{P}_{r}\left(\left\{\omega_{1}\right\}\right)=r p(q+\varepsilon-\varepsilon / p) p, & \mathbf{P}_{r}\left(\left\{\omega_{2}\right\}\right)=r p(q+\varepsilon-\varepsilon / p)(1-p), \\
\mathbf{P}_{r}\left(\left\{\omega_{3}\right\}\right)=r p(1-q-\varepsilon+\varepsilon / p) p, & \mathbf{P}_{r}\left(\left\{\omega_{4}\right\}\right)=r p(1-q-\varepsilon+\varepsilon / p)(1-p), \\
\mathbf{P}_{r}\left(\left\{\omega_{5}\right\}\right)=r(1-p)(q+\varepsilon) p, & \mathbf{P}_{r}\left(\left\{\omega_{6}\right\}\right)=r(1-p)(q+\varepsilon)(1-p), \\
\mathbf{P}_{r}\left(\left\{\omega_{7}\right\}\right)=r(1-p)(1-q-\varepsilon) p, & \mathbf{P}_{r}\left(\left\{\omega_{8}\right\}\right)=r(1-p)(1-q-\varepsilon)(1-p), \\
\mathbf{P}_{r}\left(\left\{\omega_{9}\right\}\right)=(1-r) q p q, & \mathbf{P}_{r}\left(\left\{\omega_{10}\right\}\right)=(1-r) q p(1-q), \\
\mathbf{P}_{r}\left(\left\{\omega_{11}\right\}\right)=(1-r) q(1-p)(q+\varepsilon), & \mathbf{P}_{r}\left(\left\{\omega_{12}\right\}\right)=(1-r) q(1-p)(1-q-\varepsilon), \\
\mathbf{P}_{r}\left(\left\{\omega_{13}\right\}\right)=(1-r)(1-q) p(q+\varepsilon), & \mathbf{P}_{r}\left(\left\{\omega_{14}\right\}\right)=(1-r)(1-q) p(1-q-\varepsilon) p,
\end{aligned}
$$




$$
\begin{gathered}
\mathbf{P}_{r}\left(\left\{\omega_{15}\right\}\right)=(1-r)(1-q)(1-p)(q+\varepsilon), \\
\mathbf{P}_{r}\left(\left\{\omega_{16}\right\}\right)=(1-r)(1-q)(1-p)(1-q-\varepsilon) .
\end{gathered}
$$

For example, the elementary event $\left\{\omega_{8}\right\}=\left\{\left(f_{1}, f_{4}, f_{4}, f_{4}\right)\right\} \in \mathfrak{F}$ means that the student has chosen behavior pattern $\mathrm{P}-\mathrm{AP}-\mathrm{P}$ and has failed in each of the three questions. In this case, the probability is $\mathbf{P}_{r}\left(\left\{\omega_{8}\right\}\right)=r(1-p)(1-q-\varepsilon)(1-p)$. The Kolmogorov probability model $\left(\Omega, \mathfrak{F}, \mathbf{P}_{r}(\cdot)\right)$ of the experiment $W_{r}$ constructed above enables us to write the functional relation between the environmental unit and the output pole in the form of the following two equations:

$$
\begin{gathered}
\chi_{0}\left(\omega_{1}\right)=\chi_{0}\left(\omega_{2}\right)=\cdots=\chi_{0}\left(\omega_{8}\right)=b_{1}, \\
\chi_{0}\left(\omega_{9}\right)=\chi_{0}\left(\omega_{10}\right)=\cdots=\chi_{0}\left(\omega_{16}\right)=b_{2} .
\end{gathered}
$$

The relation between the internal memory and the output pole can be found in a similar way. For example, the functional relation between the internal memory at time $\tau_{3}$ and the output pole is given by the equality $\eta_{3}\left(\omega_{s}\right)=f_{3}$ for odd $s$. These functional relations as well as those given in the sequel in fact are the random elements $\chi(\omega), \eta(\omega)$, and $\xi(\omega)$ defined pointwise. Therefore one can use the standard probability rules in order to calculate their distribution functions and conditional probabilities related to these random elements. For example,

$$
\begin{aligned}
\mathbf{P}_{r}\left(\eta_{2}=f_{3} \mid \eta_{0}=f_{1}\right) & =\mathbf{P}_{r}\left(\eta_{0}=f_{1}, \eta_{2}=f_{3}\right) / \mathbf{P}_{r}\left(\eta_{0}=f_{1}\right) \\
& =\left(\mathbf{P}_{r}\left(\left\{\omega_{1}\right\}\right)+\mathbf{P}_{r}\left(\left\{\omega_{2}\right\}\right)+\mathbf{P}_{r}\left(\left\{\omega_{5}\right\}\right)+\mathbf{P}_{r}\left(\left\{\omega_{6}\right\}\right)\right) / r \\
& =p q+p \varepsilon-\varepsilon+(1-p)(q+\varepsilon)=q .
\end{aligned}
$$

On the other hand,

$$
\begin{aligned}
\mathbf{P}_{r}\left(\eta_{3}=f_{3} \mid \eta_{0}=f_{2}\right) & =\mathbf{P}_{r}\left(\eta_{0}=f_{2}, \eta_{3}=f_{3}\right) / \mathbf{P}_{r}\left(\eta_{0}=f_{2}\right) \\
& =\left(\mathbf{P}_{r}\left(\left\{\omega_{9}\right\}\right)+\mathbf{P}_{r}\left(\left\{\omega_{11}\right\}\right)+\mathbf{P}_{r}\left(\left\{\omega_{13}\right\}\right)+\mathbf{P}_{r}\left(\left\{\omega_{15}\right\}\right)\right) /(1-r) \\
& =q+\varepsilon(1-p q)>q .
\end{aligned}
$$

This means that a learning effect occurs as a result of the exam.

In this way, the results obtained in items 2.1 2.7 can be summarized in the following theorem.

Theorem. The mathematical description of the environment, input pole, external memory, external memory information processing unit, internal memory, internal memory information processing unit, and output pole is given by the vectors $\chi(\omega), \alpha(\omega), \beta(\omega)$, $\zeta(\omega), \eta(\omega), \theta(\omega)$, and $\xi(\omega)$ on the probability space $\left(\Omega, \mathfrak{F}, \mathbf{P}_{r}(\cdot)\right)$.

The function of the exam experiment $W_{r}$ consists of students' random choice of one among two behavior patterns and their answers to three successive questions in order to obtain an objective assessment of their skills by the instructor. The final result of a student's examination produces an important effect on the attitude of the student to the instructors who take part in the examination and therefore, in one way or another, change conditions of the external environment that are not controlled.

\section{Students' optimal Behavior and solution of the Mosteller-SzéKely PARADOX}

Assume that the random event $A=\left\{\omega_{1}, \omega_{2}, \omega_{5}, \omega_{9}, \omega_{10}, \omega_{13}\right\}$ means that a student has passed the exam. Then

$$
\begin{aligned}
\mathbf{P}_{r}(A) & =\mathbf{P}_{r}\left(\left\{\omega_{1}\right\}\right)+\mathbf{P}_{r}\left(\left\{\omega_{2}\right\}\right)+\mathbf{P}_{r}\left(\left\{\omega_{5}\right\}\right)+\mathbf{P}_{r}\left(\left\{\omega_{9}\right\}\right)+\mathbf{P}_{r}\left(\left\{\omega_{10}\right\}\right)+\mathbf{P}_{r}\left(\left\{\omega_{13}\right\}\right) \\
& \left.=r\left[p q(q-p)-\varepsilon\left(1+p^{2}-p-p q\right)\right]\right)+q p(2-q)+p \varepsilon(1-q) .
\end{aligned}
$$


The problem of optimization for the experiment $W_{r}$ discussed above is to find a number $r \in[0,1]$ that satisfies the following optimality condition:

$$
\mathbf{P}_{r_{0}}(A)=\sup \left\{\mathbf{P}_{r}(A): 0 \leqslant r \leqslant 1\right\} .
$$

When determining an optimal strategy for choosing the behavior pattern in the (Mosteller) exams problem, some natural restrictions on the parameters $p, q$, and $\varepsilon$ should be taken into account, namely

$$
0<p, q<1, \quad q>p, \quad \varepsilon \geqslant 0, \quad q+\varepsilon<1, \quad q+\varepsilon-\varepsilon / p \geqslant p .
$$

The parameters $p$ and $q$ correspond to student's skills, while $\varepsilon$ is a certain learning parameter. Let us consider all possibilities that may occur to $p, q$, and $\varepsilon$ under the above restrictions.

Lemma 2. If

$$
q \geqslant(p-0.5)^{2}+0.75, \quad q+\varepsilon<1,
$$

then the optimal strategy is given by $r_{0}=1$.

Proof. Assume that

$$
q \geqslant(p-0.5)^{2}+0.75
$$

Then

$$
1-q \leqslant p(q-p)(1-p)^{-1} \leqslant p q(q-p)\left(1+p^{2}-p-p q\right)^{-1} .
$$

If $0 \leqslant \varepsilon<1-q$, then

$$
q+\varepsilon-\varepsilon p^{-1}>p, \quad p q(q-p)-\varepsilon\left(1+p^{2}-p-p q\right)>0 .
$$

The probability of the random event $A$ is equal to

$$
\mathbf{P}_{r}(A)=r\left[p q(q-p)-\varepsilon\left(1+p^{2}-p-p q\right)\right]+q p(2-q)+p \varepsilon(1-q) .
$$

This implies immediately that $r_{0}=1$.

Lemma 3. Assume that

$$
q<(p-0.5)^{2}+0.75, \quad 0 \leqslant \varepsilon<p q(q-p)\left(1+p^{2}-p-p q\right)^{-1} .
$$

Then the optimal strategy is $r_{0}=1$.

Proof. Since

$$
q<(p-0.5)^{2}+0.75
$$

the following inequalities hold:

$$
p q(q-p)\left(1+p^{2}-p-p q\right)^{-1}<p(q-p)(1-p)^{-1}<1-q .
$$

The condition

implies that

$$
0 \leqslant \varepsilon<p q(q-p)\left(1+p^{2}-p-p q\right)^{-1}
$$

$$
p q(q-p)-\varepsilon\left(1+p^{2}-p-p q\right)>0, \quad q+\varepsilon<1, \quad q+\varepsilon-\varepsilon / p>p .
$$

Therefore the optimal strategy is again $r_{0}=1$.

Lemma 4. If

$$
q<(p-0.5)^{2}+0.75, \quad \varepsilon=p q(q-p)\left(1+p^{2}-p-p q\right)^{-1},
$$

then the optimal behavior strategy is defined by the formula $0 \leqslant r_{0} \leqslant 1$.

Proof. The assumptions of Lemma 4 imply immediately that

$$
p q(q-p)-\varepsilon\left(1+p^{2}-p-p q\right)=0, \quad q+\varepsilon<1, \quad q+\varepsilon-\varepsilon / p>p .
$$

Therefore an optimal control $r_{0}$ can be taken to be $0 \leqslant r_{0} \leqslant 1$. 
Lemma 5. Assume that

$$
p q(q-p)\left(1+p^{2}-p-p q\right)^{-1}<\varepsilon \leqslant p(q-p)(1-p)^{-1}, \quad q<(p-0.5)^{2}+0.75 .
$$

Then the optimal strategy is $r_{0}=0$.

Proof. If

$$
p q(q-p)\left(1+p^{2}-p-p q\right)^{-1}<\varepsilon \leqslant p(q-p)(1-p)^{-1}, \quad q<(p-0.5)^{2}+0.75,
$$

then the following inequalities hold:

$$
p q(q-p)-\varepsilon\left(1+p^{2}-p-p q\right)<0, \quad q+\varepsilon-\varepsilon / p \geqslant p, \quad q+\varepsilon<1 .
$$

This yields $r_{0}=0$ meaning that the student chooses the behavior pattern AP-P-AP; that is, the student chooses to answer the first question to the assistant professor, the second question to the professor, and the third question again to the assistant professor.

We have determined above the sets of values of the parameters $p$ and $q$ and the region for the parameter $\varepsilon$ which enable us, in particular, to understand the independence paradox [5]. These regions correspond to the case where the students should choose the behavior pattern $\mathrm{P}-\mathrm{AP}-\mathrm{P}$ or $\mathrm{AP}-\mathrm{P}-\mathrm{AP}$ depending on the values of $p$ and $q$ used in preparing the examination, and of the learning parameter $\varepsilon$. The above argument implies that if $\varepsilon=0$, then only the behavior pattern $\mathrm{P}-\mathrm{AP}-\mathrm{P}$ should be employed given that

$$
(p, q) \in\{(p, q): 0<p, q<1, q>p\} .
$$

Observe that the optimal strategy $r_{0}=1$ for the Mosteller problem coincides with the solution of a similar problem in [4, 5] for $\varepsilon=0$.

Figure [10 consisting of four parts shows shadowed regions for the values of the parameters $p, q$ as well as those for the parameter $\varepsilon$ where the optimal plan of students' behavior at the exam should be chosen.

Let us now give a solution to the well-known independence paradox $[5$. The advice to employ only the behavior pattern $\mathrm{P}-\mathrm{AP}-\mathrm{P}$ suggested in [4, 5] is not always true. In these books, a key point in the reasoning is the necessity to provide a correct answer to the second question. Indeed, only in this case will a student have a possibility of giving two correct answers in a row which will enable him to pass the exam. In other words, a higher probability of succeeding in the second question seems to always be more essential thana lower number of questions to be answered to the professor. However, the cybernetic approach applied to this problem, as well as numerous surveys and examinations themselves, show that applications of the behavior patterns $\mathrm{P}-\mathrm{AP}-\mathrm{P}$ or $\mathrm{AP}-\mathrm{P}-\mathrm{AP}$ strongly depend on the preparation of students for examinations, as well as their capacity to learn during the exam. For students with excellent preparation whose answers will probably be independent $(\varepsilon=0)$ or satisfy the condition

$$
q \geqslant(p-0.5)^{2}+0.75
$$

the behavior pattern $\mathrm{P}-\mathrm{AP}-\mathrm{P}$ is preferable. To the contrary, for students who have weak preparation and high learning capacity, when

$$
\begin{gathered}
0<p, q<1, \quad q>p, \quad q<(p-0.5)^{2}+0.75, \\
p q(q-p)\left(1+p^{2}-p-p q\right)^{-1}<\varepsilon \leqslant p(q-p)(1-p)^{-1},
\end{gathered}
$$

the behavior pattern AP-P-AP is more reasonable.

This situationis probably true for exams taking place after 1992, not just at the N. I. Lobachevsky State University of Nizhniy Novgorod. 
$q \geqslant\left(p-\frac{1}{2}\right)^{2}+\frac{3}{4} ; 0 \leqslant \varepsilon<1-q ; r_{0}=1$

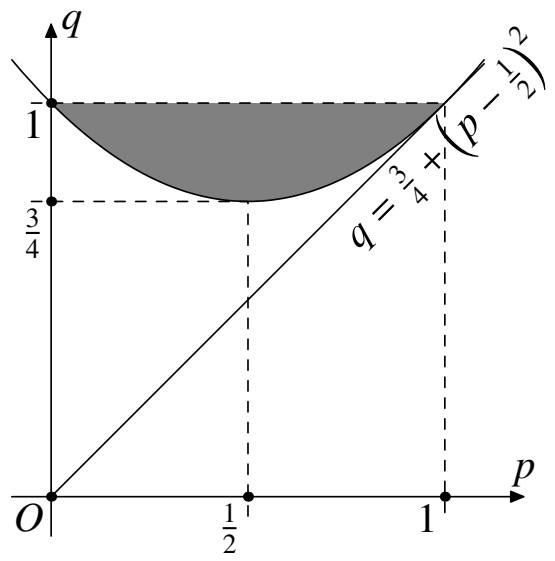

$$
q<\left(p-\frac{1}{2}\right)^{2}+\frac{3}{4}
$$

$$
\varepsilon=p q(q-p)\left(1+p^{2}-p-p q\right)^{-1}
$$

$$
0 \leqslant r_{0} \leqslant 1
$$

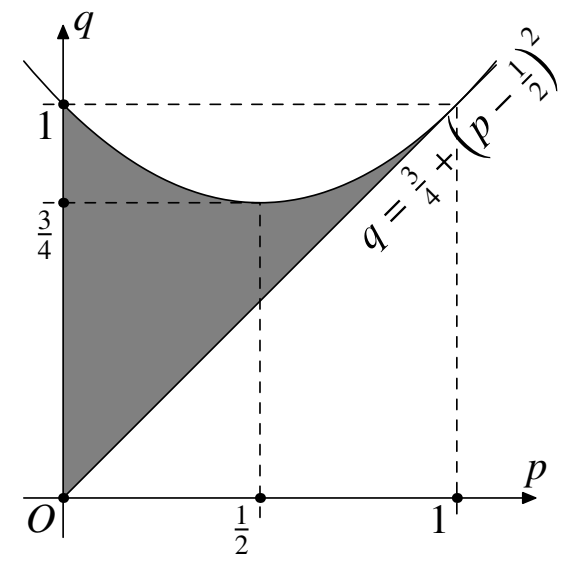

$$
q<\left(p-\frac{1}{2}\right)^{2}+\frac{3}{4}
$$$$
0 \leqslant \varepsilon<p q(q-p)\left(1+p^{2}-p-p q\right)^{-1}
$$
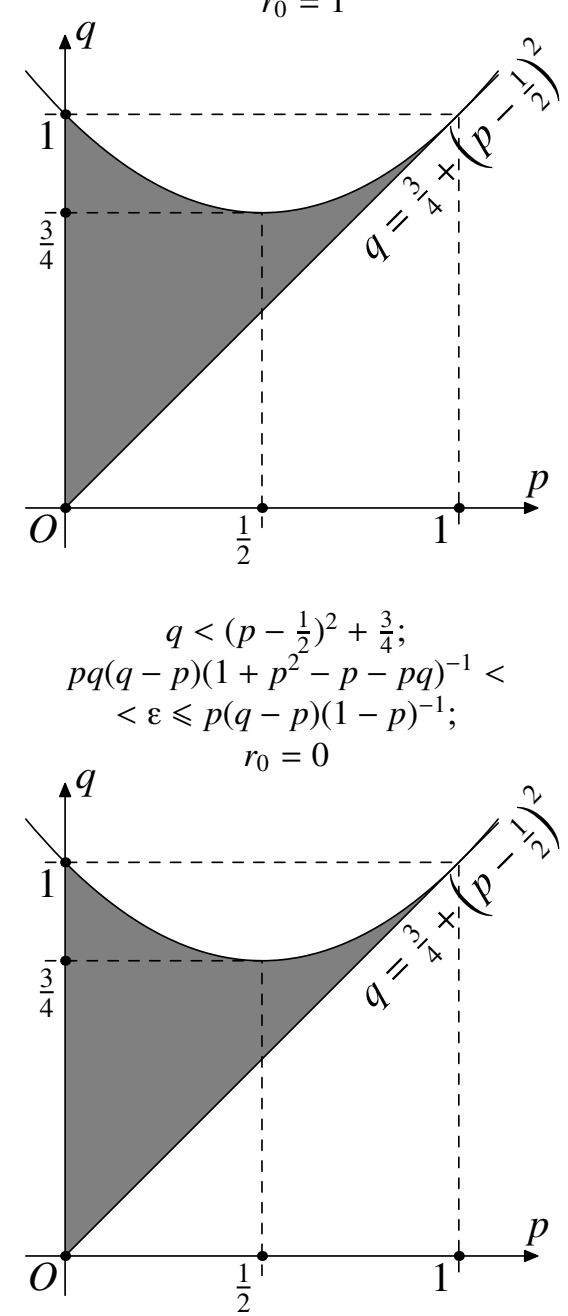

Figure 10. Regions of optimal values of $p, q$ for given values of $\varepsilon$

\section{BIBLIOGRAPHY}

1. M. A. Fedotkin, Nonlocal description of controlled stochastic problems, Mathematical Questions of Cybernetics, issue 7, "Nauka", Moscow, 1998, pp. 332-344. (Russian)

2. M. A. Fedotkin and A. M. Fedotkin, Analysis and optimization of output processes of conflicting Gnedenko-Kovalenko traffic streams under cyclic control, Avtomatika i Telemehanika (2009), no. 12, 92-108; English transl. in Automation and Remote Control (2009), no. 12, 2024-2038.

3. A. V. Zorine and M. A. Fedotkin, Optimization of control of doubly stochastic non-ordinary flows in time-sharing systems, Automation and Remote Control 7 (2005), no. 7, 1115-1124. MR 2167835

4. F. Mosteller, Fifty challenging problems in probability with solutions, Addison-Wesley, Reading, MA, 1965. MR0397810 (53:1666)

5. G. Székely, Paradoxes in probability theory and mathematical statistics, Akademiai Kiadó, Budapest, 1986. MR880020(88g:60001) 
6. A. A. Lyapunov and S. V. Yablonskiu, Theoretical problems of cybernetics, Problems of Cybernetics, issue 9, "Fizmatgiz", Moscow, 1963, pp. 5-22. (Russian)

Department of Applied Probability Theory, Faculty of Computational Mathematics and Cybernetics, N. I. Lobachevsky State University of Nizhni Novgorod, Gagarin Avenue, 23, Nizhni Novgorod, 603950, Russia

E-mail address: fma5@rambler.ru

Received 11/JUNE/2011

Translated by V. ZAIATS 